\title{
Aumento da carga de gemas da videira Sauvignon Blanc e seu efeito na interceptação
}

\section{da radiação solar e fertilidade de gemas}

\author{
Increased bud load of the Sauvignon Blanc vine and its effect on the interception of solar radiation \\ and bud fertility
}

Aumento de la carga de yemas de la vid Sauvignon Blanc y su efecto sobre la interceptación de la radiación solar y la fertilidad de las yemas

\author{
Douglas André Wurz \\ ORCID: https://orcid.org/0000-0001-6109-9858 \\ Instituto Federal de Santa Catarina, Brasil \\ E-mail: douglas.wurz@ifsc.edu.br \\ Alberto Fontanella Brighenti \\ ORCID: https://orcid.org/0000-0002-0831-4640 \\ Universidade Federal de Santa Catarina, Brasil \\ E-mail: alberto.brighenti@ufsc.br \\ Bruno Farias Bonin \\ ORCID: https://orcid.org/0000-0001-6983-2970 \\ Universidade Federal do Paraná, Brasil \\ E-mail: brunobonin@ hotmail.com \\ Ricardo Allebrandt \\ ORCID: https://orcid.org/0000-0001-8222-6254 \\ Centro Universitário Dinâmica das Cataratas, Brasil \\ E-mail: ricardoufsc@gmail.com \\ Betina Pereira de Bem \\ ORCID: https://orcid.org/0000-0003-3783-7018 \\ Vinícola Quinta da Neve, Brasil \\ E-mail: betadebem@yahoo.com.br \\ Leo Rufato \\ ORCID: https://orcid.org/0000-0001-9545-7035 \\ Universidade do Estado de Santa Catarina, Brasil \\ E-mail: leorufato@udesc.br
}

\begin{abstract}
Resumo
Buscando compreender os efeitos do aumento da carga de gemas planta ${ }^{-1}$ na interceptação da radiação solar e seu efeito na fertilidade de gemas, tem-se como objetivo desse trabalho avaliar o efeito do aumento da carga de gemas da videira 'Sauvignon em relação a interceptação da redação fotossinteticamente ativa e o seu efeito na fertilidade de gemas em região de elevada altitude de Santa Catarina. O presente trabalho foi realizado durante a safra 2016/2017, em um vinhedo comercial, localizado no munícipio de São Joaquim. Os tratamentos consistiram em quatro diferentes níveis de cargas de gemas: 15, 30, 50 e 75 gemas planta $^{-1}$. As gemas foram classificadas em férteis ou não férteis de acordo com a presença ou ausência da inflorescência. Para avaliação da interceptação da radiação fotossinteticamente ativa, utilizou-se um ceptômetro, e as medidas foram realizadas ao meio-dia durante os estádios fenológicos de plena florada, mudança de cor das bagas e maturidade. $\mathrm{O}$ aumento da carga gemas planta ${ }^{-1}$ reduz a fertilidade de gemas basais, não influenciando a fertilidade de gemas medianas e apicais da videira Sauvignon Blanc. Há uma redução da radiação fotossinteticamente ativa da videira Sauvignon Blanc em função do aumento da carga gemas planta ${ }^{-1}$, nos estádios fenológicos florada, veráison e colheita.
\end{abstract}

Palavras-chave: Vitis vinifera L.; Poda da videira; Indicação de poda.

\begin{abstract}
Seeking to understand the effects of increasing plant ${ }^{-1}$ bud load on solar radiation interception and its effect on bud fertility, the aim of this work is to evaluate the effect of increasing bud load on 'Sauvignon vines in relation to a interception of photosynthetically active writing and its effect on bud fertility in high altitude region of Santa Catarina. The present work was carried out during a 2016/2017 harvest, in a commercial vineyard, located in the municipality of São Joaquim. The treatments consisted of four different levels of bud loads: 15, 30, 50 and 75 buds plant $^{-1}$. Buds were classified as fertile or non-fertile according to the presence or absence of the inflorescence. To evaluate the interception of photosynthetically active radiation, a ceptometer was used, and measurements were taken
\end{abstract}


at midday during the phenological stages of full bloom, berry color change and maturity. The increase in plant $\mathrm{t}^{-1}$ bud load reduces the fertility of basal buds, not influencing the fertility of medium and apical buds of the Sauvignon Blanc vine. There is a reduction in the photosynthetically active radiation of the Sauvignon Blanc vine as a function of the increase in plant ${ }^{-1}$ bud load, in the flowering, verison and harvesting phenological stages.

Keywords: Vitis vinifera L.; Vine pruning; Pruning indication.

\section{Resumen}

Con el fin de comprender los efectos del aumento de la carga de yemas de la planta 1 sobre la interceptación de la radiación solar y su efecto sobre la fertilidad de las yemas, el objetivo de este trabajo es evaluar el efecto del aumento de la carga de yemas en las vides de Sauvignon en relación con la interceptación de la escritura fotosintéticamente activa y su efecto sobre la fertilidad de las yemas en la región de gran altitud de Santa Catarina. Este trabajo se llevó a cabo durante la vendimia 2016/2017, en un viñedo comercial, ubicado en el municipio de São Joaquim. Los tratamientos consistieron en cuatro niveles diferentes de carga de yemas: 15, 30, 50 y 75 yemas planta-1. Las yemas se clasificaron como fértiles o no fértiles según la presencia o ausencia de la inflorescencia. Para evaluar la interceptación de radiación fotosintéticamente activa, se utilizó un ceptómetro y se tomaron medidas al mediodía durante las etapas fenológicas de plena floración, cambio de color y madurez de la baya. El aumento de la carga de yemas de la planta 1 reduce la fertilidad de las yemas basales, sin influir en la fertilidad de las yemas medias y apicales de la cepa Sauvignon Blanc. Se produce una reducción de la radiación fotosintéticamente activa de la cepa Sauvignon Blanc en función del aumento de la carga de yemas planta-1, en las etapas fenológicas de floración, verison y cosecha.

Palabras clave: Vitis vinifera L.; Poda de vid; Indicación de poda.

\section{Introdução}

A região de elevada altitude de Santa Catarina caracteriza-se por apresentar vinhedos entre 900 e 1400 metros acima do nível do mar (Wurz et al., 2017a). Essa região apresenta uma maior disponibilidade de radiação solar e menores temperaturas noturnas na fase final de maturação. Com períodos de maturação mais longos, os vinhedos produzem uvas de maior potencial enológico (Malinovski et al., 2016). No entanto, observa-se nessa região alta disponibilidade hídrica e alto teor de matéria orgânica dos solos (Mafra et al., 2011; Bem et al., 2016), propiciando um crescimento vegetativo excessivo, baixo índice de fertilidade de gemas, afetando a produtividade e a maturação das uvas (Brighenti et al., 2014; Wurz et al., 2017b; Würz et al., 2018a).

As variações na produtividade são umas das maiores fontes de dúvidas na produção vitivinícola (Wurz et al., 2019a), sendo o aumento da carga de gemas planta ${ }^{-1}$, uma das alternativas para aumentar a produtividade dos vinhedos e melhorar o seu equilíbrio vegetativo seria através da poda de inverno (Wurz et al., 2020).

A poda invernal é uma prática realizada anualmente em regiões de clima temperado, com o principal objetivo de regular a produção temporal da videira (Miele \& Mandelli, 2012). O número de gemas que permanece nos sarmentos ou nos esporões após a poda determinará a dimensão da área foliar e o número de cachos de uvas. Vários estudos relataram diferenças, em função da intensidade e tipo de poda, no vigor vegetativo e composição final das bagas de uvas tanto para consumo in natura (Christensen et al., 1994, Ahmad et al., 2004).

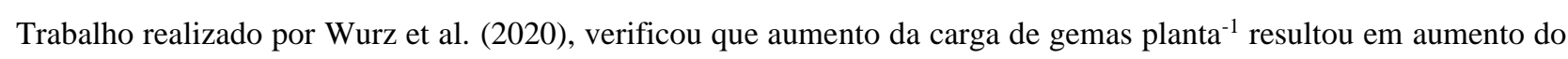
número de ramos e de cachos planta ${ }^{-1}$, resultando em aumento da produção planta e da produtividade, além de possibilitar melhor equilíbrio vegeto-produtivo, com redução do peso de poda, e obtenção do Índice de Ravaz mais adequado para a

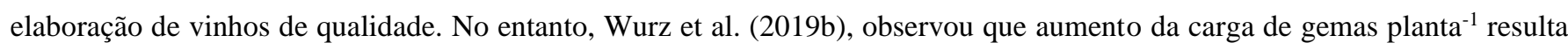
em redução da radiação fotossinteticamente ativa no dossel vegetativo da videira Cabernet Franc, ocorrendo redução da fertilidade de gemas, de acordo com Leão et al. (2017), a redução da radiação solar pode resultar em redução da fertilidade de gemas. De acordo com Jackson (2008), as condições ambientais apresentam influência na fertilidade de gemas, sendo a intensidade da luz, temperatura e disponibilidade de água, os principais fatores envolvidos. Alta intensidade da radiação solar e alta temperaturas promovem a síntese de citocininas que favorecem a diferenciação floral (Jackson, 2008).

Buscando compreender os efeitos do aumento da carga de gemas planta ${ }^{-1}$ na interceptação da radiação solar e seu 
efeito na fertilidade de gemas, tem-se como objetivo desse trabalho avaliar o efeito do aumento da carga de gemas da videira 'Sauvignon em relação a interceptação da redação fotossinteticamente ativa e o seu efeito na fertilidade de gemas em região de elevada altitude de Santa Catarina.

\section{Metodologia}

O presente trabalho caracteriza-se como pesquisa de campo, de caráter qualitativo, que foi realizado durante a safra 2016/2017, em um vinhedo comercial, localizado no munícipio de São Joaquim, coordenadas (28`17’39” S e 4955’56” O), a 1230 metros de altitude. Utilizaram-se plantas de 'Sauvignon Blanc' enxertadas sobre o porta-enxerto 'Paulsen 1103'. Os vinhedos foram implantados em 2004. O vinhedo caracteriza-se por apresentar plantas espaçadas de 3,0 x 1,5m, em filas dispostas no sentido N-S, conduzidas em espaldeira, podadas em cordão esporonado duplo, a 1,2m de altura e cobertas com tela de proteção anti-granizo, e com histórico de baixas produtividades.

Os solos da região enquadram-se nas classes Cambissolo Húmico, Neossolo Litólico e Nitossolo Háplico, desenvolvidos a partir de rocha riodacito e basalto (Santos et al., 2018). O clima da região é classificado como 'Frio, Noites Frias e Úmido’, Índice Heliotérmico de 1.714, precipitação pluvial média anual de $1.621 \mathrm{~mm}$ e a umidade relativa do ar média anual de $80 \%$ (Tonietto \& Carbonnau, 2004).

Os tratamentos consistiram em quatro diferentes níveis de cargas de gemas: 15, 30, 50 e 75 gemas planta $^{-1}$. A poda foi realizada para a 'Sauvignon Blanc' no dia 08 de setembro de 2016. Deixou-se 8, 15, 25 esporões com duas gemas cada esporão para os tratamentos 15, 30 e 50 gemas por planta, e para o tratamento 75 gemas por planta, deixou-se 30 esporões com duas gemas, e duas varas com 8 gemas cada, sendo, portanto, este tratamento podado no sistema de poda mista, caracterizada pela presença de esporões e varas. Após a realização da poda, contabilizou-se o número de gemas por planta, cegando-se as que apresentavam valores acima de 15, 30, 50 e 75 gemas planta $^{-1}$.

Para a avaliação da fertilidade de gemas, foram coletados 20 ramos de um ano, de cada tratamento, no momento de dormência profunda das plantas, após a maturação das gemas, durante o inverno de 2017. O material vegetativo dormente foi levado imediatamente a Universidade do Estado de Santa Catarina, no Centro de Ciências Agroveterinárias - Lages (SC). As gemas foram individualizadas e separadas de acordo com suas posições no ramo em gemas basais $\left(1^{\mathrm{a}}\right.$ a $\left.3^{\mathrm{a}}\right)$ e gemas medianas $\left(4^{\mathrm{a}}\right.$ a $\left.7^{\mathrm{a}}\right)$ e gemas apicais $\left(8^{\mathrm{a}}\right.$ a $\left.10^{\mathrm{a}}\right)$. Cada segmento do ramo contendo uma gema foi disposto em bandejas de isopor com espuma fenólica hidratada.

As bandejas foram colocadas em câmara com temperatura, fotoperíodo e umidade controlados (Fitotron) com $60 \%$ de umidade relativa, temperatura de $20^{\circ} \mathrm{C}$ e 14 horas de luz por dia com intensidade de 300-400 $\mu \mathrm{E} \mathrm{m}-2 \mathrm{~s} \mathrm{~s}^{-1}$ (Andreini et al., 2009). As gemas foram classificadas em férteis ou não férteis de acordo com a presença ou ausência da inflorescência.

Para avaliação da interceptação da radiação fotossinteticamente ativa, utilizou-se um ceptômetro AccuPAR (LP-80 Decagon, EUA). As medidas foram realizadas ao meio-dia, quando o sol estava no zênite, durante os estádios fenológicos de plena florada, mudança de cor das bagas e maturidade, e os resultados expressos em PAR umol m-2 s-1. As leituras foram efetuadas em três posições diferentes: na zona dos cachos, perpendicular e paralelo a linha das plantas; no dossel vegetativo, perpendicular à linha das plantas.

O delineamento experimental utilizado foi o de blocos ao acaso, com quatro blocos e cinco plantas por parcela. Os dados foram submetidos à análise de variância (ANOVA) e comparados pelo Teste Tukey a 5\% de probabilidade de erro.

\section{Resultados e Discussão}

As diferentes cargas de gemas/planta avaliadas na videira Sauvignon Blanc influenciaram a fertilidade de gemas, sendo estas avaliadas de duas formas: (a) \% de gemas férteis (Tabela 1) e (b) cachos gema ${ }^{-1}$ (Tabela 2). 
Observou-se que as diferentes cargas de gemas influenciaram a fertilidade de gemas (\% de gemas férteis) apenas para as gemas basais, e as localizadas na posição mediana e apical do ramo não foram influenciadas pelas diferentes cargas de gemas planta ${ }^{-1}$. Para as gemas medianas e apicais do ramo, observou-se uma fertilidade variando de 97,8 a 100\% (Tabela 1).

Para as gemas localizadas na posição basal do ramo, observou-se a maior fertilidade de gemas (100\%) para a carga e 15 gemas planta ${ }^{-1}$, enquanto o aumento das cargas de gemas planta ${ }^{-1}$ resultou em redução gradativa da \% de gemas férteis, com valores de 87,5, 83,5 e 80,5\% para as cargas de 30, 50 e 75 gemas planta $^{-1}$, respectivamente.

Trabalho realizado por Greven et al. (2014), sugere que ao longo dos anos, as videiras tendem a promover mudanças de seu comportamento, através de compensação do dossel vegetativo e da produtividade, principalmente em função de alterações da arquitetura de cachos e redução da fertilidade de gemas, que é um dos efeitos observados no estudo realizada na região de elevada altitude de Santa Catarina. De acordo com trabalho realizado por Wurz et al. (2019b), ao avaliar o efeito do aumento da carga de gemas planta ${ }^{-1}$ na videira 'Cabernet Franc', observou comportamento similar, com menor fertilidade das gemas basais, com aumento da carga de gemas planta $^{-1}$, sendo que essa redução da fertilidade de gemas, está associado ao maior adensamento do dossel vegetativo, reduzindo a interceptação da radiação fotossinteticamente ativa, e consequentemente reduzindo a fertilidade de gemas.

Tabela 1. Efeito da carga de gemas na fertilidade de gemas (\% gemas férteis) da videira Sauvignon Blanc (Vitis vinífera L.) em região de elevada altitude de Santa Catarina. Safra 2017.

\begin{tabular}{cccccc}
\hline Fertilidade & \multicolumn{4}{c}{ Carga de Gemas } & CV \\
\cline { 2 - 6 } (\% gemas férteis) & $\mathbf{1 5}$ & $\mathbf{3 0}$ & $\mathbf{5 0}$ & $\mathbf{7 5}$ & $\mathbf{( \% )}$ \\
\hline Gemas Basais & $100,0 \mathrm{a}$ & $87,5 \mathrm{~b}$ & $83,5 \mathrm{~b}$ & $80,5 \mathrm{~b}$ & 10,8 \\
Gemas Medianas & $100,0 \mathrm{~ns}$ & 100,0 & 97,8 & 97,8 & 3,2 \\
Gemas Apicais & $100,0 \mathrm{~ns}$ & 94,0 & 100,0 & 97,1 & 8,8 \\
\hline
\end{tabular}

*Médias seguidas da mesma letra, na linha, não diferem entre si pelo teste Tukey a $5 \%$ de probabilidade de erro.ns = não significativo pela análise de variância (ANOVA) a 5\% de probabilidade de erro. Fonte: Autores.

Observou-se efeito das cargas de gemas planta-1 para a fertilidade de gemas, avaliada através da relação do número de cachos planta-1 (Tabela 2). Assim como o observado na \% de gemas férteis, as diferentes cargas de gemas planta-1 não influenciaram o número de cachos planta-1 na posição mediana e apical dos ramos da videira Sauvignon Blanc, no entanto, observou-se efeito nas gemas localizadas na posição basal dos ramos.

$\mathrm{O}$ aumento da carga de gemas planta ${ }^{-1}$ resultou em redução do número de cachos planta ${ }^{-1}$. Enquanto a carga de 15 gemas planta ${ }^{-1}$ apresentou, em média, 1,3 cachos planta ${ }^{-1}$, para as cargas de 30, 50 e 75 gemas planta $^{-1}$, observou-se 1,0, 1,0 e 0,9 cachos planta $^{-1}$, respectivamente. Para a posição mediana e apical, observou-se variação de 1,5 a 1,8 cachos planta $^{-1}$, ou seja, observou-se um maior número de cachos planta $^{-1}$ na posição mediana e apical em relação a posição basal das gemas no ramo.

Esses resultados referentes a posição distal das gemas e sua fertilidade influenciam no sistema de poda a ser adotado, pois por apresentar uma baixa fertilidade de gemas basais, é necessário adotar um sistema de poda longa ou mista (Wurz et al., 2018b). Adaptando o tipo de poda com a posição das gemas mais férteis é possível aumentar a produtividade, pois haverá um aumento no número de gemas férteis ao adotar o sistema de poda adequado para cada cultivar (Brighenti et al., 2017; Vilar et al., 2017). 
Tabela 2. Efeito da carga de gemas na fertilidade de gemas número de cachos gema ${ }^{-1}$ ) da videira Sauvignon Blanc (Vitis vinífera L.) em região de elevada altitude de Santa Catarina. Safra 2017.

\begin{tabular}{|c|c|c|c|c|c|}
\hline \multirow{2}{*}{$\begin{array}{c}\text { Fertilidade } \\
\left(\text { Cachos gemas }^{-1}\right)\end{array}$} & \multicolumn{4}{|c|}{ Carga de Gemas } & \multirow{2}{*}{$\begin{array}{l}\mathrm{CV} \\
(\%)\end{array}$} \\
\hline & 15 & 30 & 50 & 75 & \\
\hline Gemas Basais & $1,3 \mathrm{a}$ & $1,0 \mathrm{ab}$ & $1,0 a b$ & $0,9 \mathrm{~b}$ & 16,7 \\
\hline Gemas Medianas & $1,8 \mathrm{~ns}$ & 1,7 & 1,8 & 1,5 & 8,2 \\
\hline Gemas Apicais & $1,7 \mathrm{~ns}$ & 1,6 & 1,8 & 1,6 & 12,6 \\
\hline
\end{tabular}

*Médias seguidas da mesma letra, na linha, não diferem entre si pelo teste Tukey a $5 \%$ de probabilidade de erro.ns = não significativo pela análise de variância (ANOVA) a 5\% de probabilidade de erro. Fonte: Autores.

O efeito da carga de gemas planta $^{-1}$ na interceptação da radiação fotossinteticamente ativa em diferentes estádios fenológicos está descrita na Tabela 3. Realizou-se as medições em três diferentes posições: perpendicular ao cacho, perpendicular ao dossel vegetativo e paralelo ao cacho, e observou-se efeito das cargas de gemas planta ${ }^{-1}$ na interceptação da radiação fotossinteticamente ativa nos estádios fenológicos florada, veráison e no momento da colheita.

A avaliação da interceptação da radiação fotossinteticamente ativa na posição perpendicular ao cacho apresentou comportamento semelhante nos três estádios fenológicos avaliados, observando-se maiores valores de interceptação da radiação fotossinteticamente ativa nas cargas de 15 e 30 gemas planta $^{-1}$, estas não diferentes estatisticamente entre si, enquanto as cargas de 50 e 75 gemas planta $^{-1}$ apresentaram os menores valores de interceptação da radiação fotossinteticamente ativa.

Para a posição perpendicular ao dossel vegetativo, observou-se comportamento semelhante a posição perpendicular ao cacho, onde observou-se redução da interceptação da radiação fotossinteticamente ativa ao aumentar a carga de gemas, no entanto, ressalta-se que no estádio fenológico veráison, a carga de 30 gemas planta $^{-1}$ apresentou, juntamente com as cargas de 50 e 75 gemas planta $^{-1}$, os menores valores de interceptação da radiação fotossinteticamente ativa (Tabela 3).

Quando se avaliou a interceptação da radiação fotossinteticamente ativa na posição paralela ao cacho, observou-se redução dos valores, principalmente para a carga de 75 gemas planta $^{-1}$. Os maiores valores médios de radiação solar global diária interceptada foram observados entre a floração e veráison. Neste período, uma maior quantidade de radiação solar atinge a atmosfera na latitude considerada (Vieira et al., 2011).

De acordo com Wurz et al. (2019b), ao adotar sistemas de poda com carga de gemas alta ( $>50$ gemas planta $\left.^{-1}\right)$, tornase imprescindível a adoção de técnicas de manejo com objetivo de favorecer uma melhor interceptação da radiação fotossinteticamente ativa no dossel vegetativo, como por exemplo, a escolha do tipo mais adequado da poda e a desfolha da

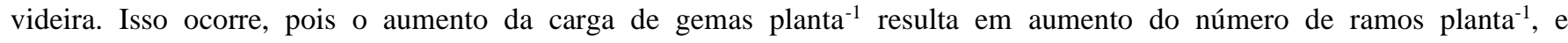
consequentemente tem-se maior adensamento do dossel vegetativo, propiciando maior sombreamento nas gemas (Wurz et al., 2020). Trabalhos realizados por Wurz et al. (2017b) e Wurz et al. (2018a), observaram que ao desfolhar a videira na região dos cachos, expondo as gemas a uma maior radiação solar, resulta em aumento da fertilidade de gemas da videira. 
Tabela 3. Efeito da carga de gemas na interceptação da radiação fotossinteticamente ativa em diferentes estádios fenológicos na videira Sauvignon Blanc (Vitis vinífera L.) em região de elevada altitude de Santa Catarina. Safra 2017.

\begin{tabular}{|c|c|c|c|c|}
\hline & \multicolumn{4}{|c|}{ Carga de Gemas } \\
\hline & 15 & 30 & $\mathbf{5 0}$ & 75 \\
\hline \multicolumn{5}{|c|}{ Plena Florada } \\
\hline Cacho (perpendicular à linha das plantas) & $204,58 \mathrm{a}$ & $36,01 \mathrm{~b}$ & $27,01 \mathrm{~b}$ & $22,68 \mathrm{~b}$ \\
\hline Dossel (perpendicular à linha das plantas) & $75,72 \mathrm{a}$ & $49,10 \mathrm{~b}$ & $27,19 \mathrm{c}$ & $24,09 \mathrm{c}$ \\
\hline Cacho (paralelo a linha das plantas) & $203,06 \mathrm{a}$ & $90,04 \mathrm{~b}$ & $86,54 b$ & $59,89 \mathrm{~b}$ \\
\hline \multicolumn{5}{|c|}{ Veráison } \\
\hline Cacho (perpendicular à linha das plantas) & $328,21 \mathrm{a}$ & $120,94 \mathrm{~b}$ & $87,97 \mathrm{~b}$ & $68,68 \mathrm{~b}$ \\
\hline Dossel (perpendicular à linha das plantas) & $148,58 \mathrm{a}$ & $43,79 b$ & $39,85 \mathrm{~b}$ & $32,36 \mathrm{~b}$ \\
\hline Cacho (paralelo a linha das plantas) & $745,09 \mathrm{a}$ & $399,52 \mathrm{ab}$ & $337,67 \mathrm{ab}$ & $211,35 \mathrm{~b}$ \\
\hline \multicolumn{5}{|c|}{ Colheita } \\
\hline Cacho (perpendicular à linha das plantas) & $152,74 \mathrm{a}$ & $120,00 \mathrm{ab}$ & $81,39 \mathrm{~b}$ & $82,21 \mathrm{~b}$ \\
\hline Dossel (perpendicular à linha das plantas) & $111,53 \mathrm{a}$ & $129,21 \mathrm{a}$ & $75,81 \mathrm{~b}$ & $71,13 \mathrm{~b}$ \\
\hline Cacho (paralelo a linha das plantas) & $87,60 \mathrm{a}$ & $76,47 \mathrm{ab}$ & $79,43 \mathrm{a}$ & $62,79 \mathrm{~b}$ \\
\hline
\end{tabular}

*Médias seguidas da mesma letra, na linha, não diferem entre si pelo teste Tukey a 5\% de probabilidade de erro.ns = não significativo pela análise de variância (ANOVA) a 5\% de probabilidade de erro. Fonte: Autores.

Ao relacionar-se os dados observados de fertilidade de gemas e radiação solar, verificou-se que o aumento da carga de gemas planta ${ }^{-1}$ resultou em redução da radiação fotossinteticamente interceptada, e reduziu a fertilidade de gemas basais. Diversos estudos citam a redução da radiação solar como principal fator responsável pela redução da fertilidade de gemas em plantas podadas com maior carga de gemas planta ${ }^{-1}$, visto que dentre os fatores que influenciam a fertilidade de gemas, destaca-se a temperatura e radiação solar (Vasconcelos et al., 2009; Greven et al., 2014). A formação da gema fértil é a consequência da diferenciação do primórdio indiferenciado em primórdio reprodutivo (Botelho et al., 2006), sendo geneticamente controlados e induzidos por vários fatores nos quais se destacam intensidade luminosa e temperaturas (Botelho et al., 2009). Condições insatisfatórias de luz durante a iniciação da inflorescência reduz severamente a fertilidade de gemas e ramos mais expostos à luz, normalmente são mais férteis (Keller \& Koblet, 1995).

\section{Conclusão}

1. O aumento da carga gemas planta $^{-1}$ reduz a fertilidade de gemas basais, não influenciando a fertilidade de gemas medianas e apicais da videira Sauvignon Blanc.

2. Há uma redução da radiação fotossinteticamente ativa da videira Sauvignon Blanc em função do aumento da carga gemas planta $^{-1}$, nos estádios fenológicos florada, veráison e colheita.

\section{Referências}

Ahmad, M. W., Junaid, M., Nafees, M., Farooq, M., \& Ali Salem, B. (2004). Effect of pruning severity on growth behavior of spur and bunch morphology of grapes (Vitis vinifera L.) cv. Perlette. International Journal of Agriculture and Biology, 6(1), 160-161.

Andreini, L., Viti, R., \& ScalabrellI, G. (2009). Study on the morphological evolution of bud break in Vitis vinifera L. Vitis, 48(4), 153-158.

Bem, B. P. de., Bogo, A., Everhart, S. E., Casa, R. T., Gonçalves, M. J., Marcon Filho, J. L., Rufato, L., Silva, F. N. da., Allebrandt, R., \& Cunha, I. C. da. (2016). Effect of four training systems on the temporal dynamics of downy mildew in two grapevine cultivars in southern Brazil. Tropical Plant Pathology, $41,370-379$.

Botelho, R. V., Pires, E. J., \& Terra, M. M. (2009). Fertilidade de gemas de videira: fisiologia e fatores envolvidos. Ambiência, 2(1), 129-144.

Brighenti, A. F. (2014). Avaliação de variedades de videira (Vitis vinifera L.) autóctones italianas no terroir de São Joaquim - SC. Tese (Doutorado em Recursos Genéticos Vegetais). Florianópolis: UFSC. 184p. 
Brighenti, A. F., Cipriani, R., Malinovski, L. I., Vanderlinde, G., Allebrandt, R., Feldberg, N. P., \& Silva, A. L. (2007). Ecophysiology of three Italian cultivars subjected to two pruning methods in Santa Catarina, Brazil. Acta Horticulturae, 1157, 381-388.

Christensen, L. P., Leavitt, G. M., Hirshfelt, D., \& Bianchi, M. (1994). The effects of pruning level and post bud break cane adjustment on Thompson seedless raisin production and quality. American Journal of Enology and Viticulture, 45(2), 141-152.

Greven, M. M., Bennett, J. S., \& Neal, S. M. (2014). of retained node number on Sauvignon Blanc grapevine vegetative growth and yield. Australian Journal of Grape and Wine Research, 20(2), 263-271.

Jackson, R. S. (2008); Grapevine structure and function In: JACKSON, R.S. (Ed.). Wine science principles and applications. 3rd ed. San Diego: Academic Press, p.50-107.

Keller, M., \& Koblet, W. (1995). Dry matter and leaf area partitioning, bud fertility and second season growth of Vitis vinifera L.: Responses to nitrogen supply and limiting irradiance. Vitis 34, 77-83.

Leão, P. C., Souza, E. M., Nascimento, J. H., \& Rego, J. I. (2017). Bud fertility of new table grape cultivars and breeding selections in the São Francisco Valley. Revista Brasileira de Fruticultura, 39(5), e-042.

Mafra, S. H. M., Cassol, P. C., MiqueluttI, D. J., ErnanI, P. R., Gatiboni, L. C., Ferreira, E. Z., Barros, M., Zalamena, J., \& Grohskopf, M. A. (2011). Atributos químicos do solo e estado nutricional de videira Cabernet Sauvignon (Vitis vinifera L.) na Serra Catarinense. Revista de Ciências Agroveterinárias, 10(1), 4453.

Malinovski, L. I., Brighenti, A. F., Borghezan, M., Guerra, M. P., Silva, A. L., Porro, D., Stefanini, M., \& Vieira, H. J. (2016). Viticultural performance of Italian grapevines in high altitude regions of Santa Catarina State, Brazil. Acta Horticulturae, 1115, 203-210.

Miele, A., \& Mandelli, F. (2012). Manejo do dossel vegetativo e seu efeito nos componentes de produção da videira Merlot. Revista Brasileira de Fruticultura, 34(4), 964-973.

Santos, H. G. dos., Jacomine, P. K. T., Anjos, L. H. C. dos., Oliveira, V. Á. De., Lumbreras, J. F., Coelho, M. R., Almeida, J. A. de., Araújo Filho, J. C. de., Oliveira, J. B. de., \& Cunha, T. J. F. (2018). Sistema brasileira de classificação do solo. (5a.ed.) rev. e ampl. Brasília: Embrapa, 356p.

Tonietto, J., \& Carbonneau, A. A. (2004). Multicriteria climatic classification system for grape-growing regions worlwide. Agricultural and Forest Meteorologv. 124, 81-97.

Vasconcelos, M. C., Greven, M., Winefield, C. S., Trought, M. C. T., \& Raw, V. (2009). Flowering process of Vitis vinifera: a review. American Journal of Enology and Viticulture, 60(4), 411-434.

Vieira, H. J., Back, A. J., Silva, A. L., \& Pereira, E. S. (2011). Comparação da disponibilidade de radiação solar global e fotoperíodo entre as regiões vinícolas de Campo Belo do Sul-SC, Brasil e Pech Rouge, França. Revista Brasileira de Fruticultura, 33(4), 1055-1065.

Vilar, P. F., Souza, E. I., Santos, L. S., Martinez, E. A., \& Ribeiro, V. G. (2017). Phytoregulators on bud fertility and cluster quality of 'Thompsom Seedles' grapes grafted onto ‘Ramsey’ Roostock. Revita Caatinga, 30(1), 97-108.

Würz, D. A., Bem, B. P., Allebrandt, R., Bonin, B., Dalmolin, L. G., Canossa, A. T., Rufato, L., \& Kretzschmar, A. A. (2017a). New wine-growing regions of Brazil and their importance in the evolution of Brazilian wine. BIO Web of Conferences, 9, 1-4.

Würz, D. A., Brighenti, A. F., Marcon Filho, J. L., Allebrandt, R., Bem, B. P., Rufato, L., \& Kretzschmar, A. A. (2017b) Agronomic performance of 'Cabernet Sauvignon' with leaf removal management in a highaltitude region of Southern Brazil. Pesquisa Agropecuária Brasileira, 52(4), 869-876.

Wurz, D. A., Allebrandt, R., Marcon Filho, J. L., Bem, B. P., Brighenti, A. F., Rufato, L., \& Kretzschmar, A. A. (2018a). Época de desfolha e sua influência no desempenho vitícola da uva 'Sauvignon Blanc' em região de elevada altitude. Revista de Ciências Agroveterinárias, 17(1), 91-99.

Wurz, D. A., Marcon Filho, J. L., Allebrandt, R., Bem, B. P., Rufato, L., \& Kretzschmar, A. A. (2018b). Reguladores de crescimento na fertilidade de gemas e maturação da cultivar 'Nebbiolo' em região de elevada altitude de Santa Catarina. Scientia Agrarian Paranaensis, 17(1), 90-95.

Wurz, D. A., Canossa, A. T., Reinher, J., Allebrandt, R., Bem, B. P., Outemane, M., Rufato, L., \& Kretzschmar, A. A. (2019a). Avaliação da fertilidade de gemas de variedades de uvas viníferas cultivadas em região de elevada altitude de Santa Catarina. Revista De Ciências Agroveterinárias, 18(1), 81-86.

Wurz, D. A., Bonin, B. F., Brighenti, A. F., Canossa, A. T., Reinher, J., Allebrandt, R., Bem, B. P., Rufato, L., \& Kretzschmar, A. A. (2019b). Efeito da carga de gemas da videira 'Cabernet Franc' na interceptação da radiação solar e na fertilidade de gemas. Revista de Ciências Agroveterinárias, 18(4), 453-458.

Wurz, D. A., Bonin, B. F., Brighenti, A. F., Canossa, A. T., Reinher, J., Allebrandt, R., Bem, B. P., Rufato, L., \& Kretzschmar, A. A. (2020). Maior carga de gemas da videira resulta em melhora dos índices produtivos e vegetativos da videira 'Cabernet Franc' cultivada em região de elevada altitude. Revista de Ciências Agroveterinárias, 19(2), 171-177. 